\title{
Channel Sharing in Multi-Hop WDM Lightwave Networks: Do We Need More Channels?
}

\author{
Srini B. Tridandapani, Member, IEEE, Biswanath Mukherjee, Member, IEEE, \\ and Geir Hallingstad, Student Member, IEEE
}

\begin{abstract}
A local lightwave network can be constructed by employing two-way fibers to connect nodes in a passive-star physical topology, and the available optical bandwidth may be accessed by the nodal transmitters and receivers at electronic rates using wavelength-division multiplexing (WDM). The number of WDM channels, $w$, in such a network is technology-limited and is less than the number of network nodes, $N$, especially if the network should support a scalable number of nodes. We describe a general and practical channel sharing method, which requires each node to be equipped with only one transmitter-receiver pair, and in which each WDM channel is shared in a time-division multiplexed fashion.

We also develop a general model for analyzing such a sharedchannel, multi-hop, WDM network. Our analysis yields a counterintuitive result: it is sometimes better to employ fewer channels than a larger number of channels. We explore bounds on the ranges of $w$ which admit queueing stability-using too few or too many channels can lead to instability. We also obtain an estimate for the optimal number of channels that minimizes network-wide queueing delay.
\end{abstract}

Index Terms - Channel optimization, channel sharing, local lightwave network, multi-hop network, passive star, performance analysis, stability, wavelength-division multiplexing.

\section{INTRODUCTION}

W AVELENGTH-DIVISION multiplexing (WDM) partitions the huge optical bandwidth into smaller manageable channels that can be accessed at electronic rates. Fig. 1 shows an optical WDM local area network (LAN) in which $N$ nodes are connected to a passive-star coupler via two-way fibers. The $N$ nodes in the system communicate with one another using tunable (wavelength-agile) or fixedtuned transmitters (lasers) and tunable or fixed-tuned receivers (filters), which may operate on one of the $w$ wavelengths, where $w \leq N$. (Fig. 1 assumes $w=N$ and each node is equipped with a tunable receiver and a fixed-tuned transmitter operating on the node's unique wavelength.) Using tunable transmitter and/or receivers, the $N$ nodes may communicate with one another directly using the single-hop approach [8].

Manuscript received June 24, 1996; revised April 8, 1997; approved by IEEE/ACM TRANSACTIONS ON NETWORKING Editor R. Ramaswami. This work was supported in part by ARPA under Contract DABT63-92-C-0031, by NSF under Grant NCR-92-05755, and by a University Research Grant from Iowa State University. A preliminary version of this paper was presented at IEEE GLOBECOM'95.

S. Tridandapani and G. Hallingstad are with the Department of Electrical and Computer Engineering, Iowa State University, Ames, IA 50011-3060 USA (e-mail: srini@ee.iastate.edu; geirhal@iastate.edu).

B. Mukherjee is with the Department of Computer Science, University of California, Davis, CA 95616 USA (e-mail: mukherje@cs.ucdavis.edu).

Publisher Item Identifier S 1063-6692(97)07372-X.

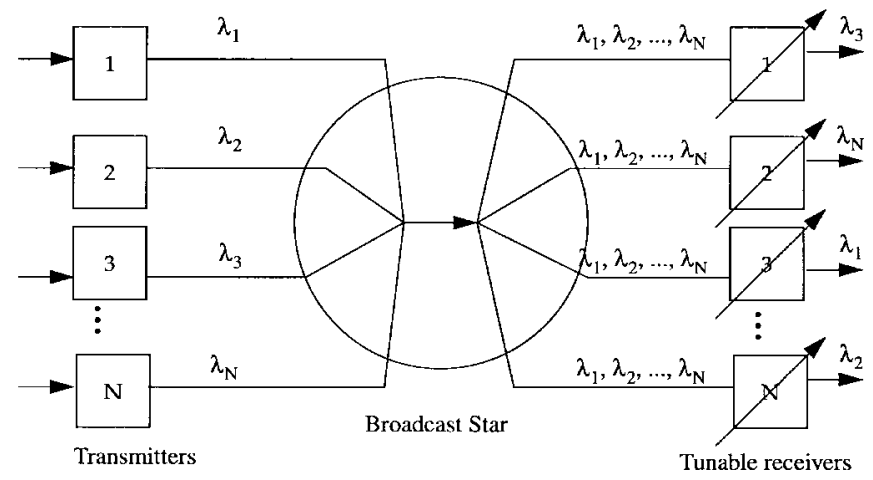

Fig. 1. A broadcast-and-select, passive-star, WDM network.

Alternatively, if all nodes are equipped with relatively inexpensive, fixed-wavelength transmitters and receivers, the $N$ nodes may communicate with one another, including nodes that are not directly connected, through the multi-hop approach [8].

In a multi-hop system, the operating wavelengths of each transmitter and receiver determine the logical topology. The degree of each node in such a logical topology depends on the number of channels in the system and the number of transceivers at each node. The degree specifies how many receiving nodes are directly connected to each transmitting node. For example, in Fig. 2(a), we show an 8-node multihop network with a nodal degree of 2. Should Node 2 desire to communicate with Node 0 , to which it is not directly connected, it would have to do so by sending information to Node 5 , using $\lambda_{2}$. Node 5 then forward this information on to Node 0 on $\lambda_{12}$. Thus, the information encounters two hops in traveling from Node 2 to Node 0 . Similarly, Node 1 is at a hop-distance of three from Node 2.

The above multi-hop technique assumes that $w$ is some integer multiple of $N$. However, this is not practical for systems with large $N$ due to various device constraints such as fiber nonlinearities and power budget [2]. Furthermore, as we will show in this paper, it may not be desirable to operate the system with $w \geq N$ channels. So, for $w<N$, we assume that each of the $w$ channels is shared by several nodes in a time-division multiplexed (TDM) fashion. ${ }^{1}$

Channel sharing in multi-hop lightwave networks has been noted by others [1], [4], [5], [7] (see [9], [10] for an exten-

\footnotetext{
${ }^{1}$ Random-access techniques, such as slotted ALOHA, may also be employed for channel sharing; however, since these techniques require more processing and cannot support high throughput, they are not considered any further.
} 


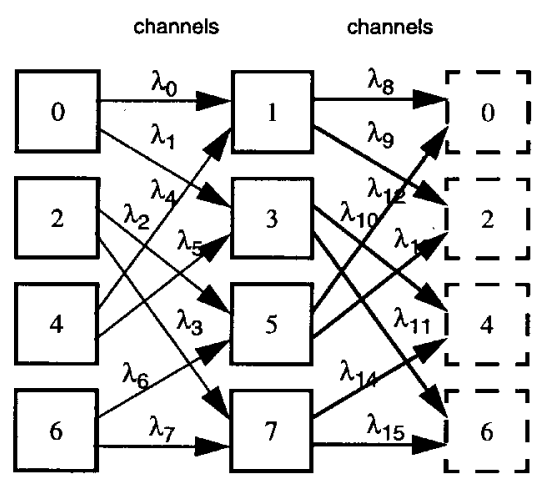

(a)

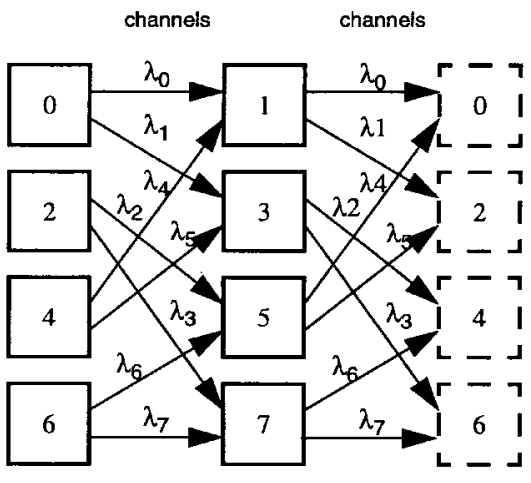

(b)

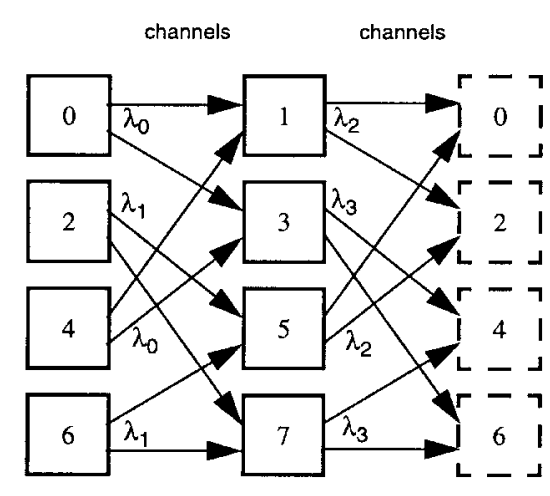

(c)

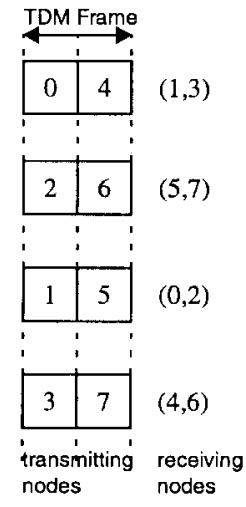

(d)

Fig. 2. (a) Logical assignment of wavelengths in an 8-node network arranged as a shufflenet with number of columns $k=2$, degree $p=2$, and $w=2 N=16$. (Note that this is also an 8-node GEMNET.) (b) Shared-channel shufflenet with $k=2, p=2$, and $w=8<2 N$. Each node has two transmitter-receiver pairs. (c) 8-node SC-GEMNET with 4 channels (each node has a single transmitter-receiver pair). (d) TDM assignment of transmitters and receivers for the SC-GEMNET shown in part (c).

sive review). Acampora [1] considered a logical topology, called shufflenet, where each node in the network has $P$ transmitter-receiver pairs. With such an arrangement, each node has a degree $P$, and networks of size $N=K P^{K}$ can be constructed with the number of channels $w=K P^{K+1}$ (without any channel sharing), where $K$ is the number of columns in the shufflenet. In Fig. 2(a), we show an 8-node network arranged as a logical shufflenet with $P=2$ and $w=16$, i.e., each communicating transmitter-receiver pair has a unique wavelength. Channel sharing may be achieved here via TDM; all receivers in a common row of the shufflenet are assigned the same wavelength. In Fig. 2(b), we consider the case where $w=8<2 N$; here, receivers in the same row are assigned the same wavelength. For example, both Nodes 2 and 3 have receivers tuned to wavelength $\lambda_{1}$. Since transmitters at both Nodes 0 and 1 are also tuned to the same wavelength, $\lambda_{1}$, these transmitters must take turns to use this channel. Any information that Nodes 0 or 1 transmit on this channel will be heard by both Nodes 2 and 3, effectively increasing the nodal out-degree of Nodes 0 and 1 . The limitation of this model is that each node requires two transmitter-receiver pairs even in the shared case. The approach that we shall present requires a single transmitter-receiver pair per node.

In [9] and [10], we proposed a method of channel sharing for the Generalized Multistage Interconnection Network (GEMNET) [6]. Our approach, called the Shared-Channel
GEMNET (SC-GEMNET), while employing a number of common features in [4], [5], [7], differs in the following ways.

- We assume that each node is equipped with only one fixed transmitter and one fixed receiver. This is costeffective and provides a basis for comparison with singlehop systems, many of which are based on a single transmitter-receiver pair per node.

- The SC-GEMNET permits the construction of networks with a wider range of sizes. That is, $N$ need not be equal to $K P^{K}$, as required in the pure shufflenet-based approach [1], or $2^{w}$, as required in the binary-hypercubebased approach [4].

- The number of wavelengths $w$ is less than $N$. The only requirement is that $N$ should be an integer multiple of $w$. Should $w>N$ channels be available, as assumed in previous studies, our analysis in this paper will still hold.

Our analysis has the following features.

- Since we assume that channel sharing is achieved through TDM, we consider fixed length packets and, therefore, use the $M / D / 1$ queueing model in our evaluation.

- Nonzero propagation delays are considered.

- The effect of channel sharing on the mean packet delay can be quantified.

- The delay expressions we derive are general and are not specific to any network architecture, such as shufflenet or GEMNET. 


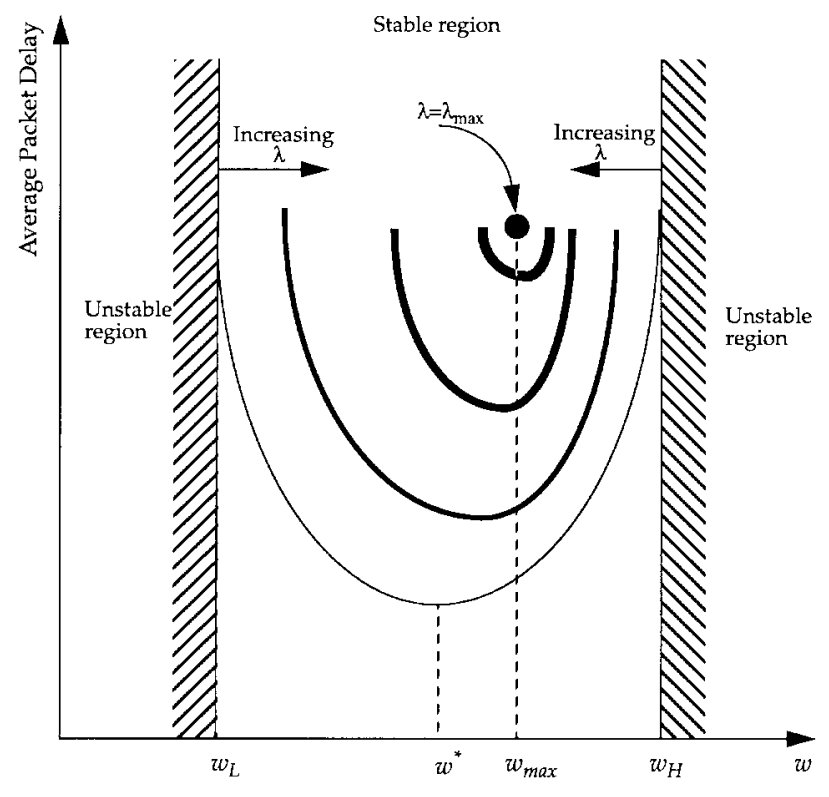

Fig. 3. Delay behavior of a shared-channel, WDM, multi-hop network with changes in load $\lambda$ and number of available wavelengths $w$.

We shall demonstrate in this paper that a multi-hop network exhibits an interesting and anomalous delay ${ }^{2}$ behavior when the number of wavelengths is varied (see Fig. 3). Within the practical region $(w \leq N)$, for small values of $w$, the average packet delay initially decreases with increasing $w$; however, for larger values of $w$, an increase in $w$ increases the delay as well. For any given load, $\lambda$, there is an optimal value of $w$ (denoted by $w^{*}$ ) for which the delay is minimized, and there are minimum and maximum values on the number of wavelengths ( $w_{L}$ and $w_{H}$, respectively) that can be effectively used. We will show that employing fewer than $w_{L}$ or more than $w_{H}$ channels leads to an unstable system. Fig. 3 also shows that, with increasing load, $w_{L}$ increases and $w_{H}$ decreases, i.e., they are collapsing toward each other, until, for a certain load $\left(\lambda_{\max }\right)$ there exists only one stable value of $w$ (denoted by $\left.w_{\max }\right)$. For the load $\lambda_{\max }, w_{L}=w_{H}=w_{\max }=w^{*}$. Characterization of this interesting behavior of shared-channel multi-hop networks is the subject of this paper.

Section II outlines our approach to constructing sharedchannel multi-hop networks and develops an analytical model to study delay performance in a generic, shared-channel, multi-hop system. Section III provides some insights into the stability and optimality of shared-channel systems, which enable us to prove that channel sharing is beneficial. Section IV concludes the paper.

\section{SC-GEMNET CONSTRUCTION AND PERFORMANCE EVALUATION}

In Fig. 2(a), we depict an 8-node 16-channel GEMNET, and in Fig. 2(c) we show the corresponding SC-GEMNET with $w=4$. The logical topology of the SC-GEMNET is equivalent to the GEMNET without channel sharing. Fig. 2(d)

\footnotetext{
${ }^{2}$ The total delay experienced by a packet is the duration between the time of its arrival at a source node and the time it is delivered to the destination, after possibly being forwarded through other intermediate nodes.
}

shows the TDM frames for the network in Fig. 2(c). From part (c) of the figure we observe that, since Node 0 has only 1 transmitter, it can only send information on $\lambda_{0}$. This information can be "heard" by both Nodes 1 and 3, since they share a channel at the receiving end. Since these two nodes have a single receiver each, the other node whose transmission they also need to hear (Node 4) must have its transmitter tuned to $\lambda_{0}$. Therefore, Nodes 0 and 4 share the transmission end of the channel. Increasing the number of nodes which share a channel at the transmission end via TDM increases queueing and frame synchronization latencies at each transmitting node. Conceptually, the capacity of a single channel is divided between two nodes, giving each node half the original capacity. However, the out-degree of these logical information streams has also been increased. This reduces the hop-distance.

We now derive an expression for the average delay encountered by a packet before it is delivered to its destination. We assume that communication is time-slotted with a slot being equal to the transmission time of a packet. All other delays in the system are normalized to this slot duration. We also note that a packet that is forwarded on a single channel may be received by multiple nodes; one of these may either "consume" the packet or forward the packet on its outgoing channel.

The following notations are used in the derivation. The number of nodes in the system is specified by $N$. The number of channels in the system, $w$, is chosen such that $N / w$ is an integer. This is to ensure fairness, and all nodes are given equal bandwidth. If $N / w$ is not an integer, fair sharing will require that some channels be wasted if each node has a single transmitter-receiver pair. Also, if $w>N$ channels are available, we assume that $w / N$ is an integer. Again, this is to guarantee fairness by distributing the available bandwidth equally among all nodes. If fixed-tuned transmitter-receiver pairs are being employed, this also implies that each node must have $w / N$ such pairs. For the rest of this derivation, we assume that $w \leq N$; results for $w>N$ can be obtained by minor modifications to the expressions we derive for $w \leq N$.

The round-trip propagation delay between a node and the passive-star coupler is denoted by $R$. We assume that all nodes are equidistant from the passive-star coupler. $F$ specifies the TDM frame length and is equal to $N / w$. A frame is comprised of $F$ slots in which $F$ different nodes get to transmit. The out-degree of a node is $P$. The out-degree is equal to $F$ for $w \leq N$, and $P=w / N$ for $w>N$.

The average rate of arrival of fresh packets per node per slot is $\lambda$. We assume that the arrival process is Poisson, and is independently and identically distributed for all network nodes. Furthermore, we assume that all packets are equally likely to be destined to any one of the network nodes except the source. Both fresh and forwarded packets arriving at a node are maintained in an infinite buffer before being processed. In order to estimate the total amount of traffic (both fresh and forwarded) presented to the network, we define the average hop-distance separating a source and destination as $\bar{h}$. Given $\bar{h}$, the average rate of packet arrivals per node per frame, including both fresh packets and forwarded packets is specified by $\lambda^{\prime}$ as $\lambda^{\prime}=\lambda N \bar{h}(1 / w)$, for $w \leq N$. 
The maximum load that a network of size $N$ can support is $\lambda_{\max }$. The maximum number of channels that can effectively support $\lambda_{\max }$ is $w_{\max }$. The minimum and maximum number of channels that can effectively support a given $\lambda$ (for $\lambda<\lambda_{\max }$ ) are $w_{L}$ and $w_{H}$, respectively, and the optimal number of channels that minimizes the average delay (defined below) experienced by a packet is $w^{*}$.

Each packet encounters the following delay components before it is delivered to its destination: 1) queueing delay at the source node and possibly at one or more intermediate nodes. This queueing delay is composed of two subcomponents: frame-synchronization delay, given by $(F-1) / 2$; and the $M / D / 1$ queueing delay, ${ }^{3}$ given by $\left.Q=\left(F \lambda^{\prime}\right) /\left[2\left(1-\lambda^{\prime}\right)\right] .2\right)$ The one-unit transmission delay along each hop. 3) Propagation delay, $R$, on each link that the packet traverses. Therefore, the total packet delay is

$$
T=\bar{h}\left[\frac{(F-1)}{2}+\frac{F \lambda^{\prime}}{2\left(1-\lambda^{\prime}\right)}+1+R\right] .
$$

For general networks, we may use the Moore bound [3] for $\bar{h}$, which is given by

$$
\bar{h}=\frac{1}{N-1}\left[\left(\sum_{i=1}^{K-1} i P^{i}\right)+K\left(N-\frac{P^{K}-1}{P-1}\right)\right]
$$

for $P>1$, and $\bar{h}=N / 2$, for $P=1$, where $K=$ $\left.\log _{P}[N(P-1)+1]-1\right\rceil$ and $P$ is the out-degree of a node. ${ }^{4}$ Equation (1) may be rewritten, in terms of $\lambda, w, R$, and $N$ as follows:

$$
\begin{aligned}
& T(N, \lambda, w, R) \\
& \quad=\bar{h}\left[\frac{\left(\frac{N}{w}\right)-1}{2}+\frac{N^{2}(\lambda \bar{h})}{2 w(w-\lambda N \bar{h})}+1+R\right] .
\end{aligned}
$$

Equation (2) indicates that, for reasonable values of $N$, the total average packet delay will increase with increasing $R$ and increasing $\lambda$. However, it is not very apparent what happens as $w$ is increased because two effects may be noticed. First, $\bar{h}$ goes up because the degree of each node is inversely proportional to $w$. Second, the frame synchronization delay decreases because the capacity available to each node on its outgoing link increases as a result of reduced sharing. The queueing delay is more difficult to characterize; we will show in Section III that the queueing initially decreases with increasing $w$ and then increases.

Also, for reasonable values of $N$ in (2), we observe that, if $\lambda$ is made arbitrarily small,

$$
\begin{aligned}
& \lim _{\lambda \rightarrow 0, R \rightarrow \infty} T(N, \lambda, w, R) \\
& =\lim _{\lambda \rightarrow 0, R \rightarrow \infty} \bar{h}\left[\frac{\left(\frac{N}{w}\right)-1}{2}+\frac{N^{2}(\lambda \bar{h})}{2 w(w-\lambda N \bar{h})}+1+R\right] \\
& \approx f(\bar{h} R) .
\end{aligned}
$$

${ }^{3}$ This queueing delay assumes that $\lambda^{\prime}$ is Poisson. Simulation results, shown later, indicate that this is a good approximation.

${ }^{4}$ The variable, $K$, represents the diameter of a Moore graph (or tree) constructed from $N$ nodes, each node having a degree of $P$. Consequently, $K-1$ represents the last complete level in the Moore graph (tree).
That is, an increase in the value of $w$ increases $\bar{h}$ but has little effect on queueing and frame synchronization latencies. Therefore, under this condition, we can expect $T$ to monotonically increase with $w$. In (2), we also note that the queueing component is $\infty$ when $w=\lambda N \bar{h}$, i.e., the system becomes unstable for

$$
\lambda>\frac{w}{N \bar{h}}
$$

Note that $\bar{h}$ itself is a function of $N$ and $w$. For small values of $w$, the numerator in the right-hand side of (4) is dominant, and for $w$ less than a certain value, denoted by $w_{L}$, the inequality in (4) is satisfied leading to an unstable system. Likewise, for larger values of $w$, the denominator becomes dominant, and for $w$ greater than a certain value, denoted by $w_{H}$, the inequality is again satisfied leading to an unstable system. This lends credibility to our qualitative description of the system's behavior in Fig. 3; further demonstration of the correctness of Fig. 3 will be provided in Section III.

We reiterate that the expressions derived above are general in that they are not restricted to any specific topology, and, therefore, provide lower bounds on the delays for any network topology. ${ }^{5}$ These definitions and expressions are used next to obtain insights into the behavior of shared-channel networks.

\section{INSIGHTS}

We will present this section in two parts. First, via a 12node example, we study the behavior of the various delay components with changes in $w$. Then, we investigate larger networks to predict the behavior of channel sharing in more realistic scenarios.

\section{A. Twelve-Node Example}

In Fig. 4, we show the various delay components as $w$ is varied in a network with $N=12, R=2$, and $\lambda=0.05$ packets/slot/node. For these parameters, if ATM cells (53 bytes) are used with each channel operating at $100 \mathrm{Mb} / \mathrm{s}$, $R=8.48 \mu \mathrm{s}$, which corresponds to a node-to-star distance of a little less than $1 \mathrm{~km}$, and the load offered by a node is 5 $\mathrm{Mb} / \mathrm{s}$ leading to a network-wide loading of $60 \mathrm{Mb} / \mathrm{s}$.

Note that, although continuous curves are shown in the figure, only the points where $N / w=\lfloor N / w\rfloor$ (or $w / N=$ $\lfloor w / N\rfloor$ ) are meaningful, because of our desire to employ a fair scheme where bandwidth is equally divided among all nodes. These valid points are highlighted on the curves. At other points, that is, when $N / w \neq\lfloor N / w\rfloor$, we have assumed that the bandwidth is equally shared by the nodes only for the sake of analysis. ${ }^{6}$

\footnotetext{
${ }^{5}$ Exact values of delay for any given network may be obtained by replacing the Moore bound in (2) with the appropriate value of the average hop-distance for the network.

${ }^{6}$ These points raise some difficulty because we originally assumed that each node has a single transmitter-receiver pair. To illustrate this difficulty consider the case where $N=12$ and $w=9$. If the available bandwidth is to be equally distributed among the 12 nodes, each node will be allocated an equivalent of 0.75 channels. Under such a condition, some of the nodes will be allocated bandwidth spread across more than one channel, implying that these nodes will need to be equipped with more than one transmitter-receiver pair. Another consequence of trying to obtain fair sharing in such a system is that the SC-GEMNET structure is no longer valid.
} 


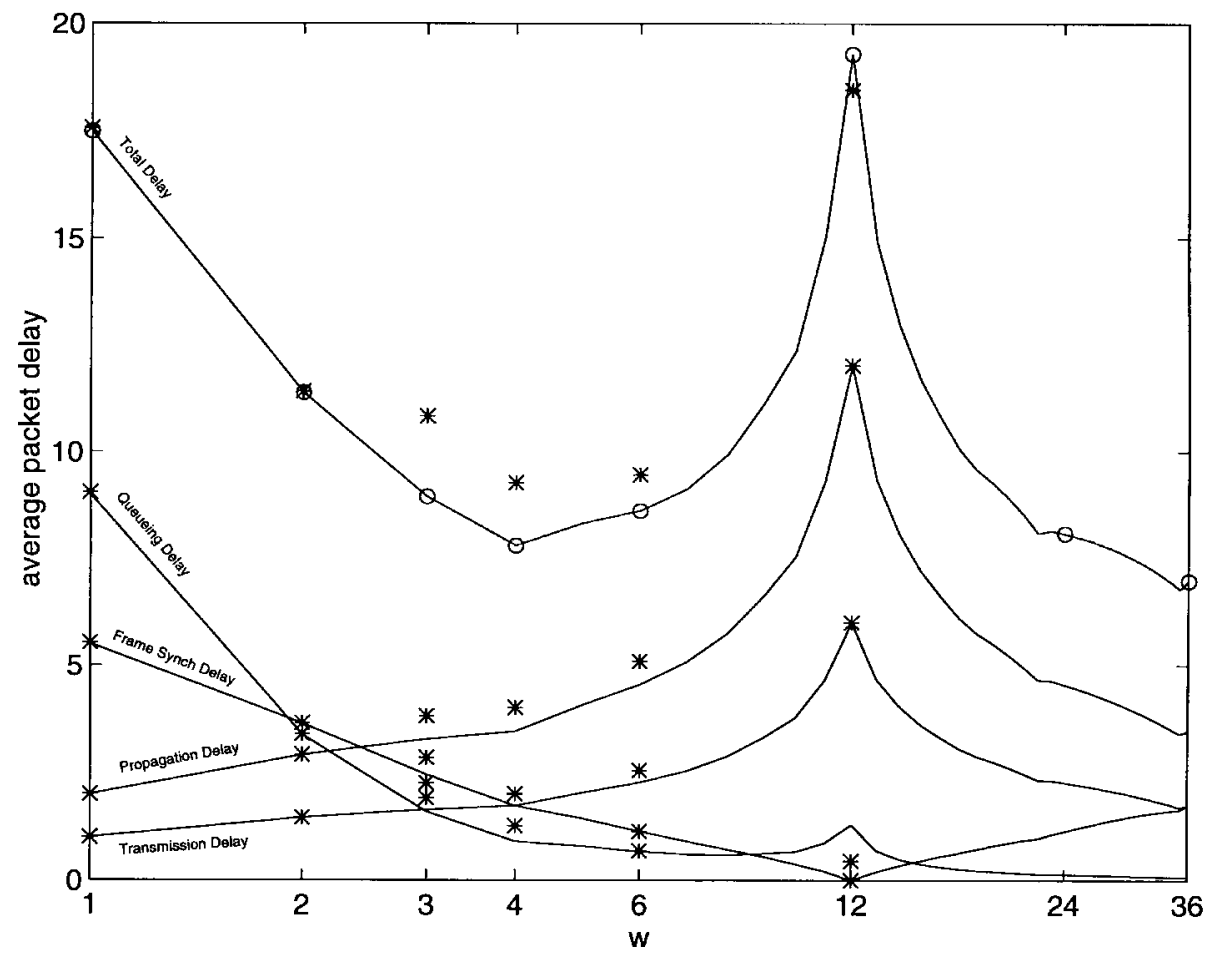

Fig. 4. Various delay components versus number of wavelengths in a 12-node network with $\lambda=0.05$ and $R=2$. The connected points are obtained from analysis. The unconnected points $(*)$ are obtained from simulations. Note that simulation points diverge from the analytical values because the analysis uses the Moore bound (lower bound on hop-distance) whereas the simulations are from SC-GEMNETS.

We first consider the case $w \leq N$ and make the following observations. We notice that, when $w$ is very small $(1$ or $2)$, the queueing delays are very large because all 12 nodes have to share the $w$ channels. For small $w$, the TDM frame synchronization delay is also significant, and we can expect this to become very large for larger values of $N$. For instance, when $w=1$, all 12 nodes are sharing a single channel (the TDM case), and the frame synchronization delay is, on average, 5.5 slots; the corresponding propagation delay is only 2 since all packets only travel across one hop. When $w=N$, each node has only one outgoing link leading to a logical ring network, and the full capacity of a link is available to each node. However, at this point, $\bar{h}=6$, leading to an average propagation delay of 12 slots. This tends to increase the average delay. Somewhere between these two extremes, the total average delay has a minimum value. Specifically, for the example network in Fig. 4, the total average delay is minimized for $w=4$.

Results from simulation of an SC-GEMNET are also plotted in Fig. 4. (The simulation points are shown with the " $*$ " symbol.) Remarkable agreement is seen when $w=1$, since this is the simple TDM case. For $w=N$, the queueing delay obtained from simulation is slightly smaller because of the smoothing effects of buffering at intermediate nodes. In between these two cases, the delay is larger because the analysis uses the Moore bound (lower bound).

In Fig. 4, we have also plotted the delay for the cases $N<w \leq 3 N$. In the figure, there are only two such points which are realizable in a fair manner: $w=24$ and $w=36$. These two cases are more expensive and require that each node have two and three transmitter-receiver pairs, respectively.
Also, for the value of $\lambda$ we are considering, we notice that the delay for the 24-channel case $(w=2 N>N)$ is higher than that for the 4-channel $(w<N)$ case. The reason is that the 4-channel case has a degree of 3, while the 24-channel case has a degree of 2 albeit with a higher capacity. Increasing $w$ further to 36 gives us the case where the degree is 3, with a larger capacity on each link of the virtual topology than in the 4-channel case. At this point, however, the delay is only slightly less than the 4-channel case. This decrease is due to the smaller queueing and synchronization delays, which result from having dedicated channels.

Thus, under certain load conditions, employing $w \leq N$ channels with a single transmitter-receiver pair per node is preferable to employing $w>N$ channels with more transmitter-receiver pairs per node. We must caution the reader, though, that under heavier load, the 36-channel system is likely to perform better. For the rest of this section, we will only study systems with a single transmitter-receiver pair per node.

An even more interesting result, as was promised in Fig. 3, can be observed in Fig. 5, wherein we depict the total delay for various loads in the same 12-node network. Here again, only those points where $N / w=\lfloor N / w\rfloor$ are meaningful, and these points are highlighted. For instance, at a load of $\lambda=0.229$, the system is unstable for all $w$ where equal sharing is possible (i.e., $w=1,2,3,4,6,12$ ) if each node has only one transmitter-receiver. For $\lambda=0.229$, stability is achieved for $w=9$ (and 10). In this case, six of the channels may be shared equally by the 12 nodes such that they each have access to a capacity equivalent to half a channel. The other channels may also be distributed equally to the 


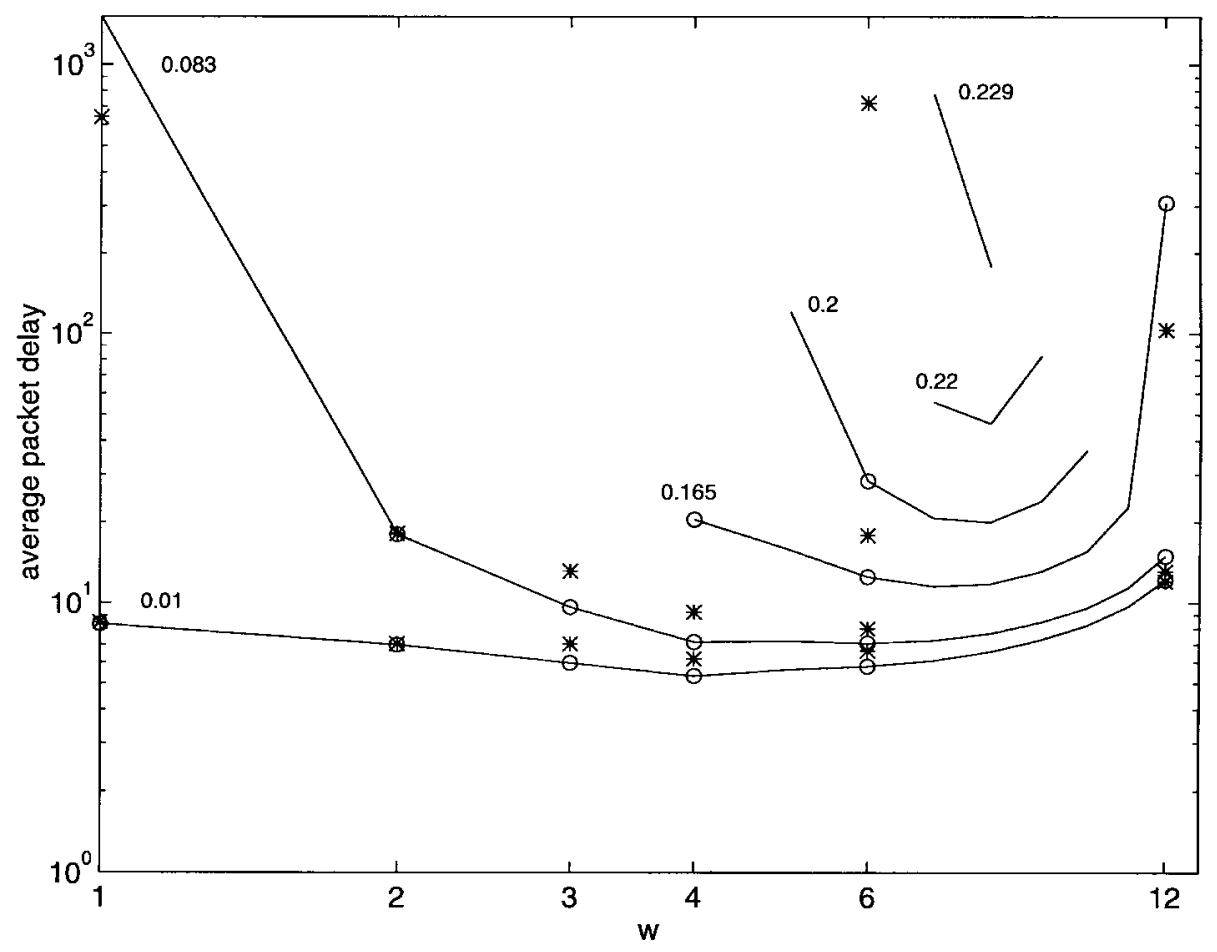

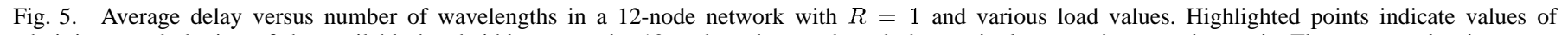
admitting equal sharing of the available bandwidth among the 12 nodes when each node has a single transmitter-receiver pair. The connected points were obtained from the analytical expressions. The unconnected points (*) were obtained from simulations.

various nodes; however, each node would then require an additional transmitter-receiver pair to utilize the additional available capacity.

When $w$ is very small, the system becomes unstable. We note that, as the load is increased, the shape of the curve becomes a more pronounced trough. For example, if $w=1$ is to be used, we have the trivial TDM case where all 12 nodes have to share the wavelength. In this case, the capacity available to each node is limited to $1 / 12=0.0833$ : that is, $\lambda>0.0833$ leads to queueing instability. On the other hand, when $w=12, \bar{h}$ is 6 , and $\lambda^{\prime} \approx 6 \lambda$, implying that the system becomes unstable for a load $\lambda>\frac{1}{6} \approx 0.1667$. The total delay is also affected by $R$ at this value of $w$.

Again, simulation results for the 12-node SC-GEMNET are provided in Fig. 5. Except for the case when $w=N$, the delay values obtained through simulation are higher than those predicted by analysis. As noted earlier, the delay is lower for the $w=N$ case because of the smoothing effects of the buffers at intermediate nodes. However, the shape of the curves is retained and the conclusions we reach are justified.

\section{B. Delay Behavior in Larger Networks}

In this subsection, we first discuss the results for $N=120$. Then we look at even larger networks. Results for $N=120$ are shown in Figs. 6 and 7, and in Table I. In Fig. 6, we show the average packet delay as a function of $w$ for various $\lambda$ and $R$. Clearly, increasing $\lambda$ or $R$ increases the delay. When $\lambda=0.05$, employing $w \leq 10$ leads to instability, confirming our prediction in Fig. 3. Note that, for large values of $R$ and small $\lambda$, the curves provide a range of possible values for $w^{*}$.
TABLE I

Optimal Number OF WAVELENGTHS, $w^{*}$, FOR VARIOUS VALUES OF $\lambda$ AND $R ; N=120$

\begin{tabular}{l|c|c|c|c}
\hline & $\lambda=0.001$ & $\lambda=0.005$ & $\lambda=0.01$ & $\lambda=0.05$ \\
\hline \hline$R=0$ & 40 & 40 & 40 & 60 \\
\hline$R=1$ & 40 & 40 & 40 & 40 \\
\hline$R=10$ & 12 & 12 & 12 & 40 \\
\hline$R=100$ & 1 & 10 & 10 & 24 \\
\hline
\end{tabular}

Particularly for the case $\lambda=0.01$ and $R=100$, there is a range of values of $w$ for which the delay, $T$, does not change appreciably. We would obviously choose $w^{*}$ from the lower end of this range.

Fig. 7 also shows the optimal number of wavelengths, $w^{*}$, that minimizes the total delay for various values of $\lambda$ and three values of $R(1,10,100)$. We note that increasing $R$ indicates a smaller $w^{*}$. For any given load, there are lower and upper bounds, $w_{L}$ and $w_{H}$, respectively, on the range of $w$ that admits stability. From the figure, it is observed that as $\lambda$ increases, $w_{L}$ needs to be larger to counter the instability resulting from increased queueing delays at each node. At the same time, $w_{H}$ needs to decrease in order to counter the queueing instability resulting from a larger number of forwarded packets. The maximum load that such a network can support, $\lambda_{\max }$, corresponds to the point where the two bounds meet, that is, at $w_{\max }$. For this example, $\lambda_{\max }=0.095$ and $w_{\max }=N / 2=60$.

We would be more interested, in general, in $w_{L}$ since this would allow us to compare our minimum requirements with technological limits. For $N=120, \lambda=0.08$ can be supported 


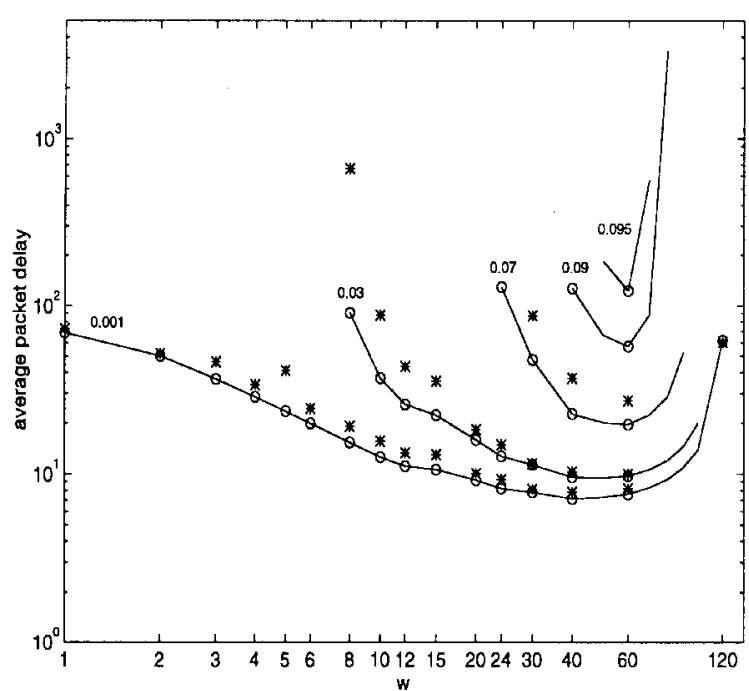

(a)

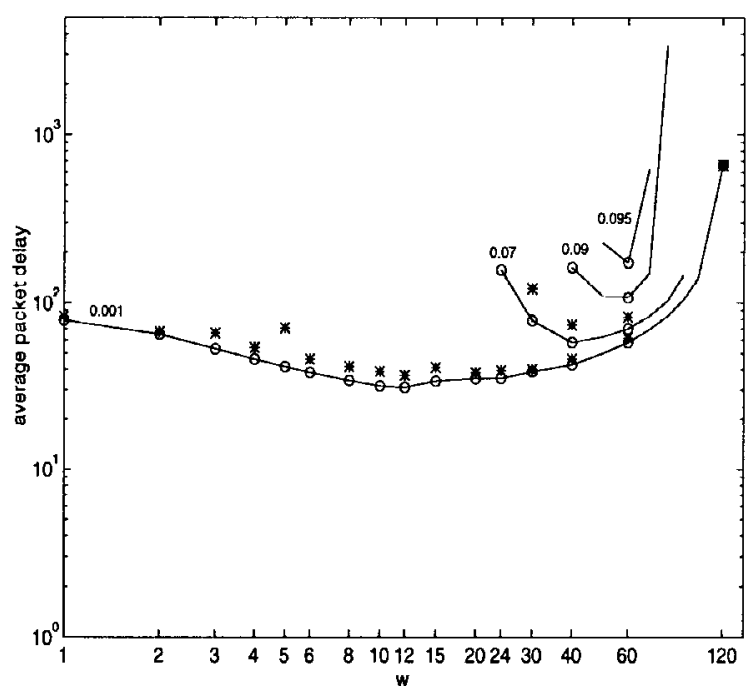

(c)

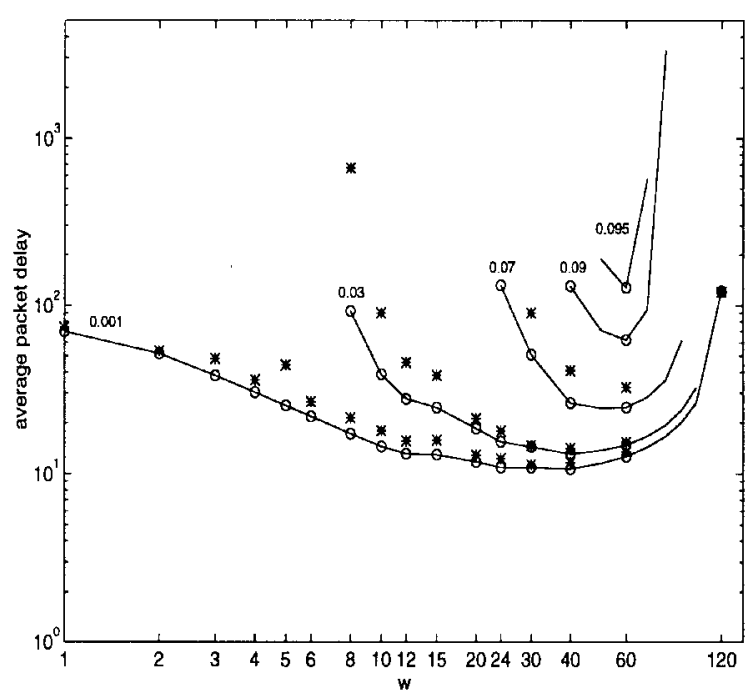

(b)

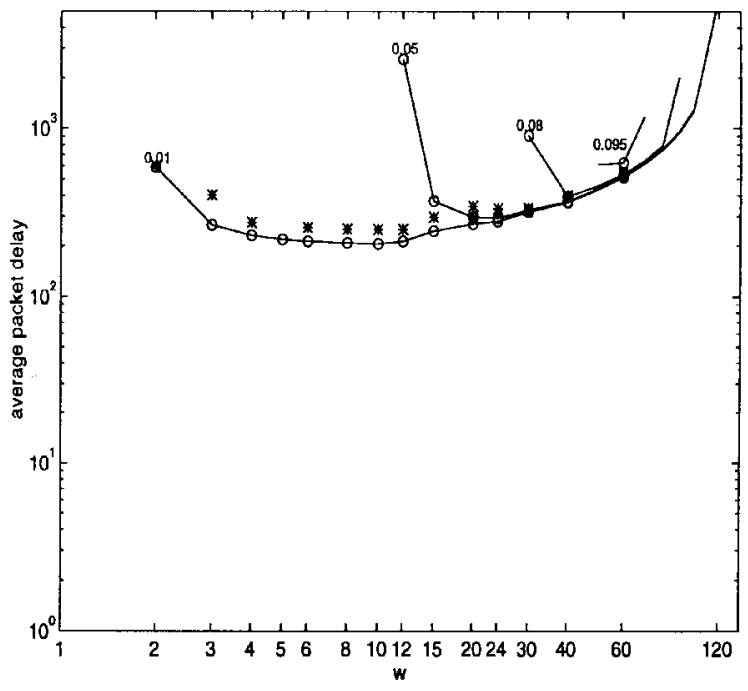

(d)

Fig. 6. Average delay, $T$, versus number of wavelengths, $w$, for various values of load in a 120-node network: (a) $R=0$; (b) $R=1$; (c) $R=10$; and (d) $R=100$.

using 30 channels. Using more than 60 channels leads to queueing instability due to forwarded traffic. We also note that $w^{*}$ is more sensitive to $R$ when $\lambda$ is small. At higher values of $\lambda$, all of the curves in the figure converge to $w=N / 2$, and $R$ does not significantly impact $w^{*}$.

In Table I, we provide values of $w^{*}$ for various values of load. The table shows that the delay can be reduced if $w$ is reduced with increasing $R$. For large $R(R=100)$, every hop implies a delay of 100 slots, so minimizing $\bar{h}$ through sharing becomes critical. Finally, in Table II, we provide values of $w^{*}, w_{L}, w_{H}$, and $w_{\max }$ for various values of $N$ and $\lambda$. The trends seem to indicate that, for small $\lambda$, the increase in $w^{*}$ is an order of magnitude smaller than the increase in $N$; for larger $\lambda$, we see that $w^{*}=w_{\max }=N / 2$ provides the best choice. ${ }^{7}$ The latter is consistent with the results in [7], where

\footnotetext{
${ }^{7}$ Some caution should be exercised in interpreting these results. We are not aware of any optical, passive-star couplers that can easily support hundreds of nodes. However, these extended, "asymptotic" results are being provided to show how channel sharing will effectively allow load management in the future, given that other technological constraints are overcome.
}

it was shown that using $w=N / 2$ maximizes the throughput. Specifically, for $N=1200$, and $\lambda=0.01$, we see that a stable system is obtained for $w$ as low as 24 . For more realistic LAN's, increasing $N$ from 60 to 120 does not increase $w_{L}$ for $\lambda \leq 0.03$. These results indicate that WDM networks are gracefully scalable: large networks do not always require an equally large number of wavelengths.

\section{CONCLUding REMARKS}

We have developed a general analytical method for modeling shared-channel, multi-hop WDM LAN's. In such networks, having a small number of channels is not only a technological limitation, but, based on our results, employing a small number of channels may actually be desirable from the system's performance point of view. When $w$ is small, channel sharing reduces hop-distance; however, making $w$ very small also leads to queueing instability since the capacity available to each transmitter is reduced. However, our intuition that a large $w$ may lead to reduced queueing is also incorrect: the increased 


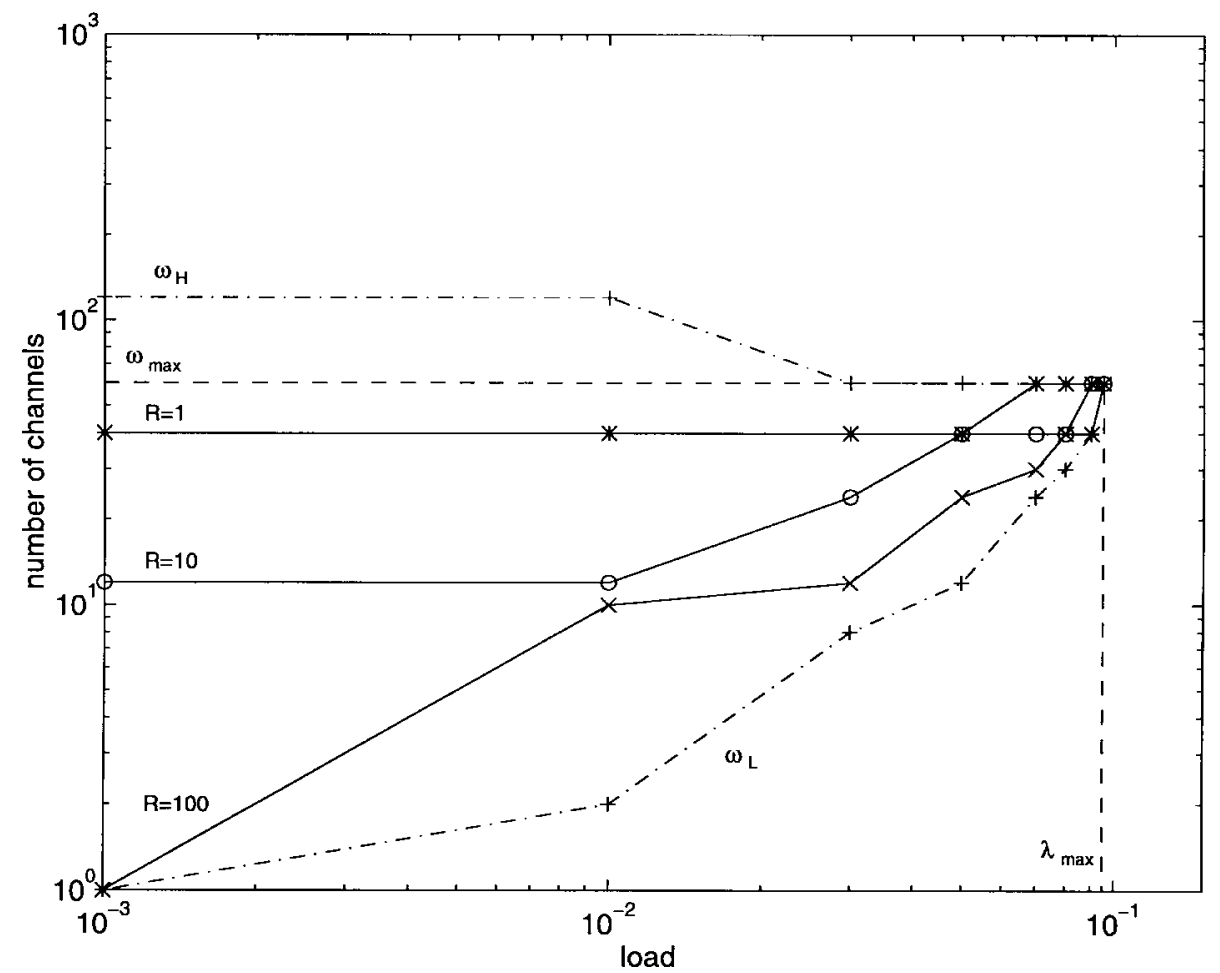

Fig. 7. Upper $\left(w_{H}\right)$ and lower $\left(w_{L}\right)$ bounds on the number of channels admitting stability and optimal number of channels $\left(w^{*}\right)$ versus load in a 120-node network.

TABLE II

$w^{*}, w_{L}, w_{H}$, AND $w_{\max }$ FOR VARIOUS $N$ AND $\lambda ; R=10$

\begin{tabular}{|c|c|c|c|c|c|c|c|c|c|c|c|c|c|c|c|c|c|c|c|c|c|c|}
\hline$\lambda$ & \multicolumn{3}{|c|}{$N=12$} & \multicolumn{3}{|c|}{$N=24$} & \multicolumn{3}{|c|}{$N=60$} & \multicolumn{3}{|c|}{$N=120$} & \multicolumn{3}{|c|}{$N=600$} & \multicolumn{3}{c|}{$N=1200$} & \multicolumn{3}{|c|}{$N=2400$} \\
\hline & $w_{L}$ & $w^{*}$ & $w_{H}$ & $w_{L}$ & $w^{*}$ & $w_{H}$ & $w_{L}$ & $w^{*}$ & $w_{H}$ & $w_{L}$ & $w^{*}$ & $w_{H}$ & $w_{L}$ & $w^{*}$ & $w_{H}$ & $w_{L}$ & $w^{*}$ & $w_{H}$ & $w_{L}$ & $w^{*}$ & $w_{H}$ \\
\hline 0.001 & 1 & 1 & 12 & 1 & 1 & 24 & 1 & 6 & 60 & 1 & 12 & 120 & 1 & 75 & 600 & 2 & 120 & 1200 & 5 & 200 & 1200 \\
\hline 0.010 & 1 & 1 & 12 & 1 & 4 & 24 & 2 & 6 & 60 & 2 & 12 & 120 & 12 & 75 & 300 & 24 & 120 & 600 & 48 & 300 & 1200 \\
\hline 0.030 & 1 & 1 & 12 & 1 & 6 & 24 & 3 & 6 & 60 & 8 & 24 & 60 & 50 & 120 & 300 & 120 & 300 & 600 & 300 & 600 & 1200 \\
\hline 0.053 & 1 & 4 & 12 & 2 & 6 & 24 & 6 & 15 & 30 & 15 & 40 & 60 & 120 & 200 & 300 & 300 & 400 & 600 & 1200 & 1200 & 1200 \\
\hline 0.055 & 1 & 4 & 12 & 2 & 6 & 24 & 10 & 15 & 30 & 20 & 40 & 60 & 150 & 200 & 300 & 400 & 400 & 600 & & & \\
\hline 0.060 & 1 & 4 & 12 & 3 & 6 & 24 & 10 & 15 & 30 & 20 & 40 & 60 & 200 & 300 & 300 & 600 & 600 & 600 & & & \\
\hline 0.068 & 1 & 4 & 12 & 3 & 6 & 24 & 10 & 15 & 30 & 24 & 40 & 60 & 300 & 300 & 300 & & & & & & \\
\hline 0.070 & 1 & 4 & 12 & 3 & 6 & 24 & 10 & 20 & 30 & 24 & 40 & 60 & & & & & & & & & \\
\hline 0.080 & 1 & 4 & 12 & 4 & 6 & 24 & 12 & 20 & 30 & 30 & 40 & 60 & & & & & & & & & \\
\hline 0.090 & 2 & 4 & 12 & 4 & 6 & 12 & 15 & 30 & 30 & 40 & 60 & 60 & & & & & & & & & \\
\hline 0.095 & 2 & 4 & 12 & 4 & 8 & 12 & 15 & 30 & 30 & 60 & 60 & 60 & & & & & & & & & \\
\hline 0.100 & 2 & 4 & 12 & 6 & 8 & 12 & 20 & 30 & 30 & & & & & & & & & & & & \\
\hline 0.110 & 2 & 4 & 12 & 6 & 8 & 12 & 30 & 30 & 30 & & & & & & & & & & & \\
\hline 0.120 & 2 & 4 & 12 & 6 & 12 & 12 & 30 & 30 & 30 & & & & & & & & & & & & \\
\hline 0.140 & 3 & 4 & 12 & 8 & 12 & 12 & & & & & & & & & & & & & & & \\
\hline 0.150 & 3 & 4 & 12 & 12 & 12 & 12 & & & & & & & & & & & & & & & \\
\hline 0.190 & 4 & 6 & 6 & & & & & & & & & & & & & & & & & \\
\hline 0.220 & 6 & 6 & 6 & & & & & & & & & & & & & & & & & \\
\hline$w_{\max }$ & 6 & & & 12 & & & 30 & & & 60 & & & 300 & & 600 & & 1200 \\
\hline
\end{tabular}

hop-distance arising from using many dedicated channels increases queueing delays, due to an increase in forwarded traffic, even to the point of instability. It appears, therefore, that there is an optimal number, $w^{*}<N$, of channels that should be used for systems with a single transmitter-receiver pair per node. The results indicate that $w^{*}$ is far less than linear in its relationship with $N$ for low load conditions; for saturating loads $w^{*}=N / 2$ minimizes delay. This indicates that channel 
sharing allows WDM LAN's to gracefully scale-up with future advances in both technology (increment in available $w$ ) and demand (increment in $N$ ). Additionally, we have shown in [9] and [10] that sharing can provide considerable benefits in the case of multicast traffic, by reducing the number of forwarded packets.

\section{REFERENCES}

[1] A. S. Acampora, "A multichannel multihop local lightwave network," in Proc. IEEE GLOBECOM'87, Nov. 1987.

[2] C. A. Brackett, "Dense wavelength division multiplexing networks: Principles and applications," IEEE J. Select. Areas Commun., vol. 8, pp. 947-964, Aug. 1990

[3] W. G. Bridges and S. Toueg, "On the impossibility of directed Moore graphs," J. Combinatorial Theory, series B, vol. 29, pp. 339-341, 1980.

[4] P. W. Dowd, "Wavelength division multiple access channel hypercube processor interconnection," IEEE Trans. Computers, vol. 41, pp. 1223-1241, Oct. 1992

[5] M. Hluchyj and M. J. Karol, "Shufflenet: An application of generalized perfect shuffles to multihop lightwave networks," in Proc. IEEE INFOCOM'88, Mar. 1988, pp. 379-390.

[6] J. Iness, S. Banerjee, and B. Mukherjee, "GEMNET: A generalized, shuffle-exchange-based, regular, scalable, and modular multihop network based on WDM lightwave technology," IEEE/ACM Trans. Networking, vol. 3, pp. 470-476, Aug. 1995.

[7] M. Kovacevic, M. Gerla, and J. Bannister, "On the performance of shared-channel multihop lightwave networks," in Proc. IEEE INFOCOM'95, June 1995, pp. 544-551.

[8] B. Mukherjee, "WDM-based local lightwave networks, Part I: Singlehop systems, Part II: Multi-hop systems," IEEE Network, vol. 6, pp. 12-27, May 1992; and vol. 6, pp. 20-32, July 1992.

[9] S. Tridandapani and B. Mukherjee, "Multicast traffic in multi-hop lightwave networks: Performance analysis and an argument for channel sharing," in Proc. IEEE INFOCOM'96, Apr. 1996, pp. 345-352.

[10] "Channel sharing in multi-hop WDM lightwave networks: Realization and performance of multicast traffic," IEEE J. Select. Areas Commun., vol. 15, pp. 488-500, Apr. 1997.

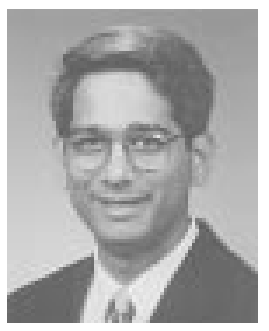

Srini B. Tridandapani (S'86-M'95) received the B.E. degree from Anna University, Madras, India, in 1988 and the Ph.D. degree in electrical engineering from University of Washington, Seattle, in 1994.

He was a Post-Doctoral Researcher/Lecturer in Computer Science at the University of California Davis during the 1994-1995 academic year. In 1995, he joined the Iowa State University as an Assistant Professor of Electrical and Computer Engineering. In Fall of 1997, he will join the University of Michigan Medical School. His research interests include the design and analysis of high-speed networks, particularly optical networks, parallel and distributed systems, and fault-tolerant systems. He is interested in applying advances in these technologies to medical information systems.

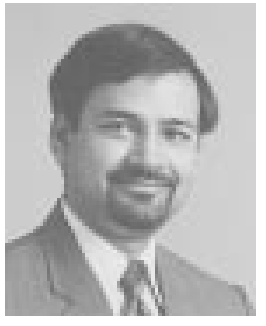

Biswanath Mukherjee (S'82-M'87) received the B.Tech. (Hons) degree from Indian Institute of Technology, Kharagpur, India, in 1980 and the Ph.D. degree in electrical engineering from University of Washington, Seattle, in June 1987.

At Washington, he held a GTE Teaching Fellowship and a General Electric Foundation Fellowship. In July 1987, he joined the University of California, Davis, where he has been Professor of Computer Science since July 1995, and Chairman of Computer Science since September 1997. His research interests include lightwave networks, network security, and wireless networks.

Dr. Mukherjee is co-winner of paper awards presented at the 1991 and the 1994 National Computer Security Conferences. He serves on the editorial boards of the IEEE/ACM TRANSACTIONS ON NETWORKING, IEEE Network, and Journal of High-Speed Networks. He served as the Technical Program Chair of the IEEE INFOCOM'96 Conference.

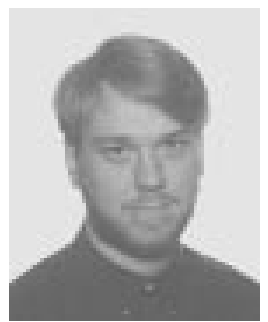

Geir Hallingstad (S'95) received the B.S. and M.S degrees, both in computer engineering, from Iowa State University in 1996 and 1997, respectively.

His research interests include design and analysis of lightwave networks and broadband networks supporting multimedia applications. 